\title{
El suicidio consumado en el Partido Judicial de Huelva.
}

\author{
Consummated suicide in the legal jurisdiction of Huelva.
}

\section{JL. Romero Palanco ${ }^{1}$ y JJ. Gamero Lucas ${ }^{1}$}

\section{RESUMEN}

Se da cuenta de la incidencia del suicidio consumado en el Partido Judicial de Huelva, durante el año 200l, para el que se obtiene una tasa de 8.66 suicidios por cada 100.000 habitantes, con una proporción de 2.4 hombres por cada mujer.

Se analiza el estado civil de los suicidas de uno y otro sexo, así como la edad media de los mismos, encontrándose diferencias significativas de esta entre los hombres (39.6 años), frente al colectivo de mujeres (65.2 años). Se estudia la distribución de los suicidios en relación con los meses del año y con los días de la semana, alcanzándose una mayor incidencia durante el segundo trimestre del año y, globalmente, durante los fines de semana.

La ahorcadura es el mecanismo más frecuentemente empleado (41..8\%), a expensas solo de los varones, seguido de la precipitación (23.53\%) y del empleo de tóxicos (17.65\%). El 58.8\% de los suicidas tenían antecedentes psiquiátricos, habiendo sido diagnosticados de síndrome depresivo en el $\mathbf{9 0 \%}$ de los casos. El $\mathbf{2 9 . 4 \%}$ de los suicidas habían hecho intentos previos de suicidio, habiendo sido estos repetidos en más de dos ocasiones en casi la mitad de los casos.

Palabras clave: Medicina Legal. Patología forense. Suicidio consumado.

Cuad Med Forense 2005; 11(39):5-9

\section{ABSTRACT}

In this paper we report the incidence of suicide committed in the Legal Jurisdiction of Huelva during the year 2001. The rate of suicides per 100.000 inhabitants was 8.66 with a proportion of 2.4 men for every woman.

The marital status of those of either sex who committed suicide is analyzed, as well as their average age. Significant differences of age was found between men (39.6) and women (65.2). The distribution of suicides by month and day of the week is also analyzed, the greatest incidence of suicides occurring during the second quarter of the year and, in general, at the weekends.

Hanging, the most employed method (4I. I 8\%), was chosen exclusively by men, followed by jumping from a height (23.53\%) and the use of toxic substances (17.65\%). $58.8 \%$ of those who committed suicide had a psychiatric history, having been diagnosed a depressive syndrome in $90 \%$ of the cases. $29.4 \%$ of suicide victims had made previous suicide attempts and in half of these cases the number of attempts had been more than two.

Key words: Legal Medicine. Forensic Pathology. Consummated suicide.

Correspondencia: José-Luis Romero Palanco. Facultad de Medicina. Universidad de Cádiz. Plaza Fragela s/n. 11003 Cádiz. Tel.: 9560151 90, Fax: 9560151 91, E-mail: joseluis.romero@uca.es.

1 Cátedra de Medicina Legal.- Facultad de Medicina.- Universidad de Cádiz. 


\section{INTRODUCCIÓN:}

El análisis de las muertes de etiología suicida ha venido siendo objeto de nuestra consideración en publicaciones anteriores [I], [2], [3], [4], en las cuales nos hemos ocupado de la incidencia de este tipo de muertes en la ciudad de Sevilla (desde 1953 a 1987) y en la población de la provincia de Cádiz.

Pese a que el estudio del suicidio puede llevarse a cabo desde muy diferentes puntos de vista, en el presente trabajo, al igual que en los anteriores, nos centraremos en realizar un estudio descriptivo del suicidio consumado en el Partido Judicial de Huelva. Debemos resaltar que hasta el momento de realizar el presente estudio se carecía de datos que nos permitieran conocer la tipología de esta variedad de muerte violenta en Huelva.

\section{MATERIAL Y MÉTODOS:}

El estudio del suicidio consumado en el Partido Judicial de Huelva se ha realizado sobre los que se produjeron durante el año 200I, que supuso un total de diecisiete casos. Dicho Partido Judicial está integrado por siete poblaciones (Aljaraque, Beas, Gibraleón, Punta Umbría, San Bartolomé de la Torre, San Juan del Puerto y Trigueros), además de la ciudad de Huelva, con un total de 196.282 habitantes.

A efectos de unificar la recogida de datos, se utilizó el mismo modelo de protocolo o encuesta, que habíamos utilizado en otros estudios similares, el cual contempla, entre otros, datos relativos a la edad, sexo, estado civil, nivel sociocultural y situación laboral de los suicidas. Asimismo, se recogen datos relativos al acto suicida, tales como la fecha, la hora, día de la semana, lugar donde se lleva a cabo el suicidio y el mecanismo por el que este se consuma. También incluye la recogida de datos relativos a la existencia de intentos previos de suicidio y de antecedentes de interés psiquiátrico.

Los datos antes reseñados se obtuvieron a partir de los existentes en los diferentes Juzgados de Primera Instancia e Instrucción. Tras la búsqueda inicial en los libros de registro, para identificar las diligencias judiciales incoadas con ocasión de este tipo de muertes, se completó la recogida de los datos consultando directamente en las mismas.

\section{RESULTADOS OBTENIDOS:}

I) Tasas de suicidios.- La tasa encontrada fue de 8.66 suicidios por cada 100.000 habitantes, la cual se encuentra muy por encima de la que con anterioridad habíamos encontrado en la provincia de Cádiz (4.78 y 3.70 para los años 1999 y 2000, respectivamente) y por encima también de las cifras que se dan para España en su globalidad que, según CORBELLA [5], viene a estar en torno a 5 suicidios por cada 100.000 habitantes y año.

2) Sexo de los suicidas.- El $70.59 \%$ de los suicidas eran hombres y el $29.41 \%$ mujeres, lo que representa una proporción de 2.4 hombres por cada mujer, cifra esta sensiblemente inferior a los valores encontrados en la provincia de Cádiz (4.52 hombres por cada mujer), pero muy próxima a las encontradas en Asturias por HINOJAL [6] (2.5 hombres por cada mujer), en Barcelona por MARTÍ AMENGUAL y CORBELLA [7] (2.33 hombres por cada mujer) y en Bilbao por ENTRENA y ALCARAZ [8] (2.23 hombres por cada mujer). 
3) Estado civil.- Analizada la muestra en su totalidad, la mayor parte de los suicidas eran casados (35.29\%), seguido de los solteros (23.53\%). Al diferenciar por sexos, las mujeres suicidas en su mayoría eran casadas (40\%) o viudas (40\%). Los varones ofrecían porcentajes iguales para los casados y los solteros (33.3\%).

4) Edad media y sexo.- Existen diferencias altamente significativas en cuanto se refiere a la edad media de uno y otro sexo, siendo notoriamente superior la edad media en el caso de las mujeres (65.2 años), frente a la obtenida para los hombres (39.6 años). Estos resultados vienen a coincidir, en términos generales, con los de otras zonas geográficas de nuestro país, en las que la edad media de las mujeres es superior a la de los hombres, aunque sin llegar a alcanzar diferencias tan extremas.

5) Días de la semana y época del año.- Al igual que en el estudio llevado a cabo entre la población de la provincia de Cádiz, la mayor incidencia del suicidio se produjo en sábado (23.5\%), siendo de resaltar que si también se consideran los producidos en domingo, el 41 . $18 \%$ de los suicidios se produjeron en estos dos días.

Por lo que concierne al mes del año, la mayor incidencia de suicidios tuvo lugar durante el mes de junio (17.65\%). Distribuidos los suicidios atendiendo a trimestres naturales, los mayores porcentajes se obtuvieron en los meses de abril, mayo y junio, con un total del $35.29 \%$ de los suicidios.

6) Mecanismos de muerte.- El mecanismo más frecuentemente empleado por los suicidas fue la ahorcadura (41. I 8\%), seguido de la precipitación (23.53\%) y de la ingestión de tóxicos (17.65\%). Este orden de frecuencias viene a coincidir con las observaciones realizadas en la población de la provincia de Cádiz, las cuales incluyen amplias zonas rurales. Por el contrario, en los estudios llevados a cabo en Sevilla, Bilbao y Barcelona, los cuales se centran en zonas urbanas, el medio preferentemente empleado fue la precipitación, seguido de la ahorcadura (Sevilla y Bilbao) o de la ingestión de tóxicos (Barcelona).

Otros procedimientos empleados en el Partido Judicial de Huelva, aunque en menor medida, fueron las armas de fuego (5.88\%), arrojarse al paso del tren (5.88\%) y las quemaduras (5.88\%). Es de resaltar la ausencia de casos de suicidio mediante la asfixia por sumersión, circunstancia esta que también hemos observado en el estudio llevado a cabo entre la población de la provincia de Cádiz.

7) Mecanismos de muerte y sexo.- Al analizar los procedimientos utilizados por los suicidas atendiendo al sexo de estos, se aprecian diferencias significativas (Tabla I). En el caso de los hombres, el procedimiento más comúnmente empleado es la ahorcadura (58.33\%), seguido de la precipitación (16.67\%), siendo utilizados con menor frecuencia las armas de fuego (8.33\%), la ingestión de tóxicos (8.33\%) y arrojarse al paso del tren (8.33\%). En el caso de las mujeres, los métodos de elección fueron la precipitación (40\%) y la ingestión de tóxicos (40\%), seguido de las quemaduras. Estos resultados son similares a los encontrados en la población de la provincia de Cádiz. Tal como se muestra en la Tabla II, existen diferencias significativas en la edad media calculada para cada uno de los mecanismos de muerte. 
Tabla I: Mecanismos de muerte y sexo.

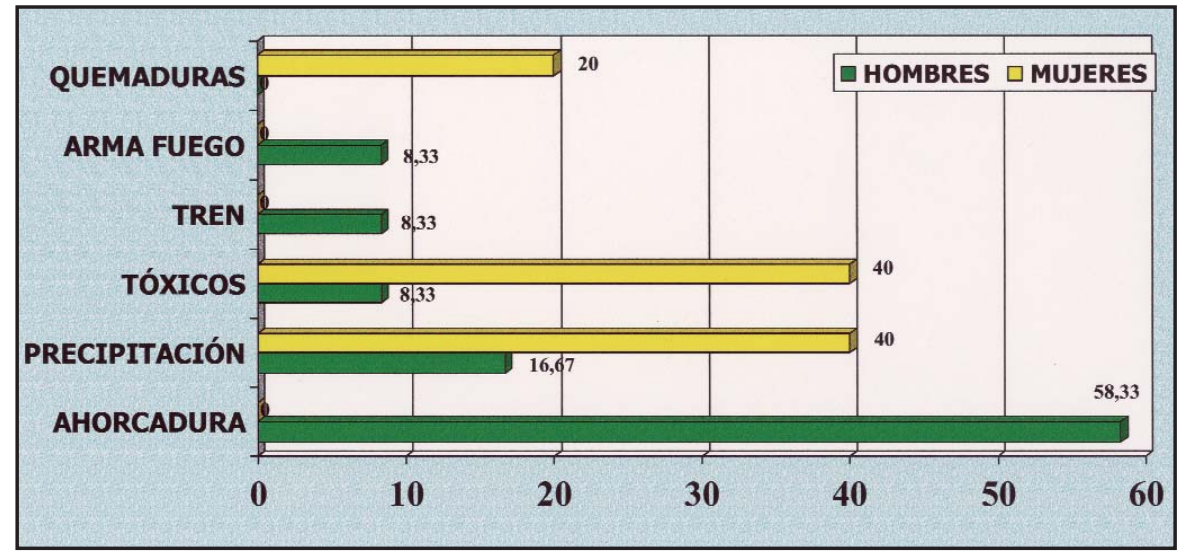

Tabla II: Mecanismos de muerte y edad media.

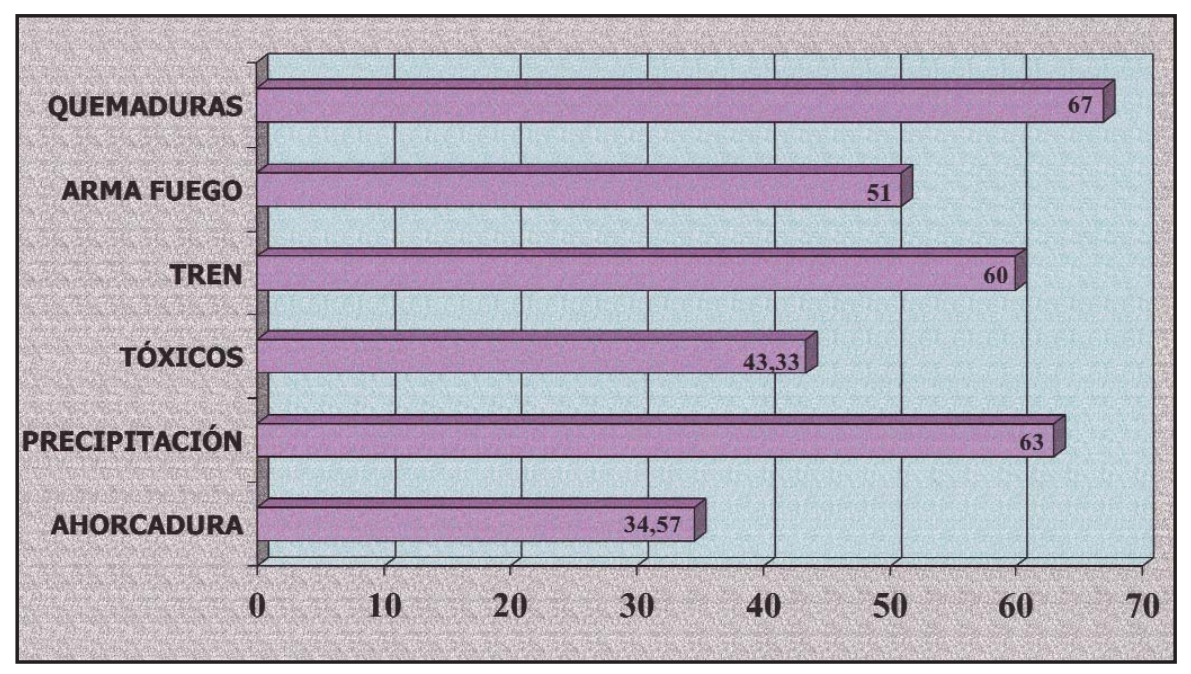

8) Antecedentes psiquiátricos e intentos previos de suicidio.- Como ya hemos indicado en otras ocasiones, pese a que no participamos plenamente de las teorías de DELMAS [9], quien sostenía que todos los suicidios eran patológicos, no siendo otra cosa que una manifestación más de la enfermedad mental, hemos estimado pertinente recoger en nuestro modelo de encuesta o protocolo, diferentes aspectos relativos a la existencia de antecedentes personales de interés psiquiátrico y de intentos previos de suicidio, a fin de aproximarnos a la influencia real que las enfermedades mentales pueden tener en el desencadenamiento de los actos suicidas.

Llama la atención que en más de la mitad de los casos (58.8\%), los suicidas tenían antecedentes personales de interés psiquiátrico, de forma similar a lo que ocurre en la provincia de Cádiz (45.74\%). La frecuencia de los mismos es variable al correlacionarlos con los diferentes mecanismos de muerte, alcanzándose los mayores valores en los casos de 
suicidio mediante la ingestión de tóxicos (100\%), seguido de los casos de precipitación (75\%) y de ahorcadura (42.86\%), como se muestra en la Tabla III. No se toman en consideración el resto de los mecanismos, dado el escaso volumen de la muestra estudiada.

Tabla III: Antecedentes de carácter psiquiátrico.

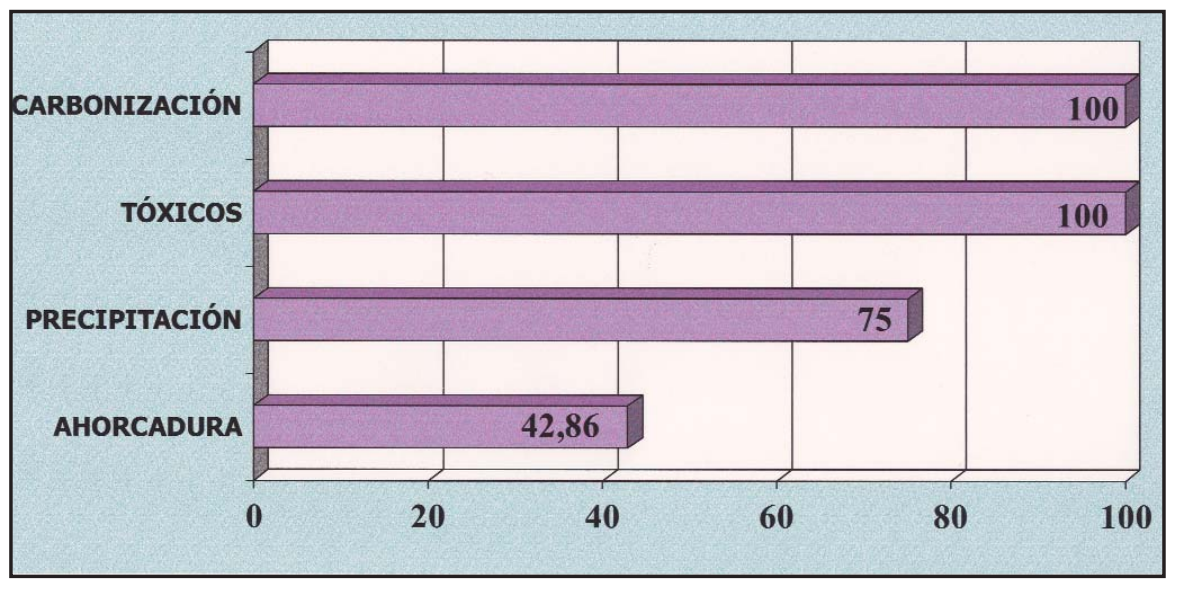

Asimismo, el 29.4\% de los suicidas habían hecho intentos previos de suicidio, habiendo sido estos repetidos en más de dos ocasiones en casi la mitad de los casos. Los intentos previos de suicidio se identificaron en el grupo de suicidas que consumaron este mediante la precipitación, la ingestión de tóxicos y la bonzonización, no habiéndose registrado ningún caso de intento previo de suicidio entre los que llevaron a cabo el suicidio mediante la ahorcadura.

Analizada la muestra en relación con los antecedentes de interés psiquiátrico, el síndrome depresivo constituye la patología más frecuentemente encontrada, alcanzando al $90 \%$ de los suicidas. El 10\% restante corresponde a cuadros de adicción a drogas.

Señalemos, por último, que todos los que habían realizado intentos previos de suicidio tenían antecedentes psiquiátricos de carácter depresivo.

\section{BIBLIOGRÁFÍA:}

I.- Romero Palanco JL. Aspectos epidemiológicos del suicidio en la ciudad de Sevilla, referidos a los años de 1953 a 1977. Rev Esp Med Legal. 1985; 42/43: 35-54.

2.- Romero Palanco JL, Gamero Lucas J, Fernández Muñoz J. El suicidio consumado en la ciudad de Sevilla, en los años de 1978 a 1982.- Actas de las VII Jornadas Internacionales de Medicina Legal. Sevilla. 1986. 3.- Romero Palanco JL, Gamero Lucas J, Vizcaya Rojas MA, Arufe Martínez MI, Hernández Triviño A. Evolución del suicidio consumado en la ciudad de Sevilla en los años de 1978 a 1987. Rev Esp Med Legal. 1989; 58/59: 9-21.

4.- Romero Palanco JL, Gamero Lucas J. Suicidio consumado en la provincia de Cádiz, en el bienio 1999-2000.- Comunicación presentada en las IXO Jornadas de la Sociedad Española de Medicina Legal y Forense. Madrid. 2001.
5.- Corbella Corbella J: El suicidio. En: Gisbert Calabuig JA. Medicina Legal y Toxicología. 50 edición. Editado por Masson S.A. Barcelona, 1998. pp 276-284.

6.- Hinojal Fonseca R: El suicidio en un área asturiana. Rev Esp Med Legal. 1978; 50/5I: 66-69.

7.- Martí Amengual G, Corbella Corbella J. Evolución del suicidio consumado en Barcelona. Monografías Médicas Jano. 1988; 9: 27-31.

8.- Entrena Ruiz-0gario J], Alcaraz Manzano R. Estudio del fenómeno suicida en el área del Gran Bilbao y zonas adyacentes, durante los años 1985-1986. Rev Esp Med Legal. 1987; 50/51:17-25.

9.- Delmas A: Psychologie pathologique du suicide. 10 ed. Edit. Alcan. Paris, 1932. 